\title{
Parametric imaging with contrast enhanced ultrasound: Usefulness for characterization of dynamic effects of microvascularization for hepatocellular carcinoma and focal nodular hyperplasia
}

Shu-Guang Zhenga,b,1,*, Hui-Xiong Xu ${ }^{\mathrm{a}, \mathrm{b}}$, Lin-Na Liu ${ }^{\mathrm{a}, 1}$, Yan Wang ${ }^{\mathrm{b}}$, Yi-Feng Zhang ${ }^{\mathrm{a}}$, Le-Hang Guo ${ }^{\mathrm{a}}$, Chang Liu ${ }^{\mathrm{a}}$, Jun-Mei Xua ${ }^{\mathrm{a}}$, Li-Ping Sun ${ }^{\mathrm{a}}$ and Jian $\mathrm{Wu}^{\mathrm{a}}$ ${ }^{a}$ Department of Medical Ultrasound, Tenth People's Hospital of Tongji University, Shanghai, China ${ }^{\mathrm{b}}$ Department of Medical Ultrasonics, The First Affiliated Hospital, Sun Yat-Sen University, Guangzhou, China

[Clinical Hemorheology and Microcirculation 55(3) (2013), 375-389]

DOI: $10.3233 / \mathrm{CH}-121652$

URL: http://iospress.metapress.com/content/x278rt37022p5831

Due to a typesetting error "Shu-Guang Zheng" was wrongly indicated as the corresponding author. The correct corresponding author should be "Hui-Xiong Xu".

The correct footnote to this article should read:

*Corresponding author: Hui-Xiong Xu, Department of Medical Ultrasound, Tenth People's Hospital of Tongji University, No. 301, Yanchangzhong Road, Shanghai 200072, China. E-mail: xuhuixiong@ hotmail.com. 